Published as: Shonin, E., Van Gordon, W., \& Griffiths M.D. (2014). Does mindfulness meditation have a role in the treatment of psychosis? Australian and New Zealand Journal of Psychiatry, 48, 124-127.

\title{
Do mindfulness-based therapies have a role in the treatment of psychosis?
}

Edo Shonin ${ }^{1,2 *}$ (Research Psychologist), William Van Gordon ${ }^{1,2}$ (Research Psychologist) and Mark D. Griffiths ${ }^{1}$ (Professor)

${ }^{1}$ Psychology Division, Nottingham Trent University, Nottinghamshire, UK, NG1 4BU

${ }^{2}$ Awake to Wisdom Centre for Meditation, Mindfulness and Psychological Wellbeing, Nottingham, UK

*Corresponding author. Division of Psychology, Chaucer Building, Nottingham Trent University, Burton Street, Nottingham, UK, NG1 4BU. Tel: +44 (0)115 8484460. Email: meditation@ntu.ac.uk

Words $=2,375$

Key Words: Psychosis, Schizophrenia, Meditation, Mindfulness, Behavioural interventions 


\section{Do mindfulness-based therapies have a role in the treatment of psychosis?}

\section{Introduction}

Mindfulness is a form of meditation that originates from Buddhist practice and was first introduced into Western clinical settings in the 1970s. The practice of mindfulness is fundamentally concerned with developing and open and unbroken awareness of present moment cognitive-effective and sensory experience. According to Shonin et al (2013a), mindfulness effectuates a greater perceptual distance from distorted cognitive and affective processes and this meta-awareness facilitates the regulation (i.e., via the non-reactive observance) of habitual maladaptive responses.

During the last two decades, a credible evidence base has emerged supporting the utilisation of mindfulness meditation in the treatment of mood and anxiety disorders, and a mindfulness interventional approach (known as Mindfulness Based Cognitive Therapy [MBCT]) is now advocated by the National Institute for Health and Clinical Excellence [NICE] and the American Psychiatric Association for the treatment of specific forms of depression. Accordingly, in recent years, clinical attention has begun to focus on the utility of mindfulness for treating other psychopathologies including psychotic disorders.

Psychotic experiences are typified by different degrees of reality distortion as well as deficits in orientating response (e.g., circumstance, place, and identity) and perceptual skills (Chadwick et al., 2005). Meditation is a subtle process in which, whether effectuated by direct means (i.e., meditative analysis) or indirect means (i.e., present-moment observance), the meditation practitioner is ultimately compelled to question not only the nature of reality, but also their ontological stance (i.e., how, or even whether the 'self' actually exists) (Shonin et al., 2013b). Given that this subtle process can be extremely challenging and confusing even 
for people of "healthy" clinical status, the question arises whether it is prudent to utilise a meditation-based recovery model for people with psychosis. To date, empirical evidence relating to this question has differed considerably. This paper provides: (i) a concise review and appraisal of empirical findings supporting or opposing the operationalisation of mindfulness for the treatment of psychotic disorders, (ii) a discussion of whether mindfulness should be deployed as a treatment for psychosis, and (iii) recommendations for practice and research.

\section{Literature search}

A literature search using MEDLINE, Science Direct, PsychInfo, and Google Scholar electronic databases was undertaken for papers published up to June 30, 2013. The search criteria employed were 'meditation', OR 'mindfulness' AND 'psychosis', OR 'psychotic', OR 'schizo*'. Included studies were those published in refereed academic journals that reported a clinical and/or empirical assessment (quantitative or qualitative) of the effects of mindfulness meditation on psychosis-related outcomes in participants with or without a history of psychiatric illness. Studies where the meditation technique was not clearly specified but appeared to be similar to mindfulness were also included. However, studies of interventions (e.g., Acceptance and Commitment Therapy) that integrate mindfulness meditation as an adjunct to more traditional dialogue-based therapeutic techniques were excluded. Likewise, studies of meditation techniques that are not explicitly mindfulnessbased (e.g., Transcendental Meditation, Qigong, etc.) were excluded.

\section{Psychosis induced by meditation}

An early study by Walsh and Roche (1979) reported three cases of individuals with a history of schizophrenia that, whilst participating in meditation retreats, experienced acute psychotic 
episodes. Garcia-Trujillo et al (1992) described a further two cases of acute psychosis precipitated by meditation in individuals previously diagnosed with schizotypal personality disorder. A further three cases of individuals with a psychiatric history presenting with meditation-induced psychotic symptoms were reported by Chan-ob and Boonyanaruthee (1999). Yorston (2001) illustrated the case of a 25-year old female graduate student in which delusional episodes accompanied by both violent outbursts and inappropriate laughter were precipitated by meditation. Psychotic episodes induced by meditation were also reported in two individuals without a history of psychiatric illness (Sethi and Subhash, 2003). More recently, the case of a male patient who developed an acute and transient psychosis with polymorphic symptomatology following meditation was also reported (Kuijpers et al., 2007).

These abovementioned case study findings appear to suggest that caution may be warranted in the meditation-based treatment of psychotic disorders. However, outcomes should be considered in light of the many limitations of these studies including the fact that: (i) they all utilised low participant number uncontrolled designs, (ii) most of the participants had a history of psychiatric illness, (iii) participants had invariably been exposed to intensive meditation practise (i.e., involving up to 18 hours of meditation per day with prolonged periods of fasting and/or silence), (iv) most of the studies provided inadequate information regarding the full range of meditation techniques employed, and (v) participants were attending open meditation retreats rather that practicing meditation within a clinical framework setting.

\section{Psychosis treated by meditation}

The first study to utilise mindfulness meditation as the primary mechanistic agent for treating psychosis was conducted by Chadwick et al (2005). Participants $(n=11)$ with unremitting psychotic experiences that were diagnosed with a schizophrenia-spectrum disorder attended 
90-minute weekly group mindfulness sessions for six weeks. The sessions comprised a tenminute guided mindfulness exercise in conjunction with psychoeducation and guided discovery components. Lengthy periods of silence were avoided and all participants received an audiotape of guided meditations. Significant pre-post improvements were demonstrated in general clinical functioning and participants' ability to respond mindfully to distressing thoughts and images increased by $36.6 \%$. These clinical gains were replicated in a small $(n=$ 22) randomised controlled trial (RCT - with wait-list control) of a ten-week mindfulness intervention (Chadwick et al., 2009).

A further small-scale wait-list controlled RCT $(n=23)$ was conducted by Langer et al (2012). Patients with a schizophrenia-spectrum disorder received an eight-week mindfulness intervention (weekly sessions of 60-minute duration) and a CD of guided meditations was provided to facilitate daily self-practice. The mindfulness group demonstrated significant improvements over controls in their ability to respond mindfully to distressing thoughts and images.

More recently, a larger-scale RCT was conducted by Chien and Lee (2013). They assessed the effectiveness of a mindfulness-based psychoeducation program for Chinese outpatients ( $n$ =96) with schizophrenia. Compared to a treatment-as-usual control group, patients who received the mindfulness intervention demonstrated significant long-term improvements in psychotic symptom severity, psychosocial functioning, and frequency of rehospitalisation. Another recent (uncontrolled design) study found that patients $(n=16)$ recovering from their first psychotic episode demonstrated significant reductions in agoraphobic symptoms and psychoneuroticism following completion of a four-week (twice-weekly) mindfulness-based intervention (MBI) (van der Valk et al., 2013). 
Utilising the same intervention protocol as Chadwick et al's (2005) aforementioned pilot study, Abba et al (2008) conducted a grounded theory analysis of the treatment experiences of schizophrenia patients $(n=16)$ with prominent positive symptoms. Mindfulness was shown to improve delusion-related regulatory capacity and modify participant's perception of psychotic experiences via a three-stage process: (i) becoming centred and stable in the awareness of psychotic experiences, (ii) allowing psychotic episodes to come and go without attempting to modify them, and (iii) empowerment through acceptance and non-judgemental awareness of self and symptoms.

A further qualitative study analysed the experiences of outpatients $(n=15)$ with co-occurring schizophrenia and anxiety who had recently completed an eight-week mindfulness intervention (Brown et al., 2010). Participants attended weekly classes of one-hour duration and participant numbers were limited to five participants per cohort. Participants practiced meditation for an average of 25 minutes per day and attended $88 \%$ of classes. Analysis of semi-structured interviews yielded themes of increased relaxation capacity, reductions in psychiatric (including psychotic) symptoms, and cognitive-attentional changes.

Ashcroft et al (2012) conducted a qualitative study to assess the suitability of an MBI as an early intervention for psychosis. Participants $(n=9)$ with persistent positive symptoms and/or anxiety symptoms attended weekly one-hour sessions that included a ten-minute guided meditation. Participants found the mindfulness intervention to be acceptable and reported experiencing increased levels of self-acceptance. Participants also felt more empowered and in control when they stopped trying to avoid or modify psychotic symptoms. These findings were consistent with those of two small-scale feasibility studies ( $n=8$ [Jacobsen et al., 2011]; $n=2$ [Taylor et al., 2009]), and were replicated in a more recent small-scale qualitative 
investigation involving outpatients $(n=3)$ experiencing distressing auditory hallucinations (Dennick et al., 2013).

Although the aforementioned studies in this section indicate that mindfulness meditation appears to ameliorate psychotic symptoms, the generalisability of findings is limited by factors such as: (i) small sample sizes, (ii) poorly defined or inactive control conditions (i.e., there was no control for potential confounding factors such as therapeutic alliance, etc.), (iii) intervention heterogeneity (i.e., there were differences in overall treatment duration, facilitator contact hours, etc.), and (iv) fidelity of implementation and/or adherence to practice were not assessed. Furthermore, considering there is currently debate regarding how to define the mindfulness construct, variations are likely to exist in terms of how different mindfulness-based therapies (MBTs) teach and operationalise mindfulness (Shonin et al., 2013a). An aspect of this debate relates to whether or not contemporary mindfulness therapies continue to embody the 'essence' of mindfulness practice as per its traditional Buddhist construal. In other words, it should be acknowledged that there important differences between the more traditional contextualisation of mindfulness and its use within mindfulness-based therapies (MBTs). Accordingly, for a given patient population, treatment outcomes for individual MBTs (e.g., MBSR) may not necessarily generalise to other MBTs, nor to more traditional forms of mindfulness meditation.

\section{The utility of mindfulness for the treatment of psychosis?}

Based on the studies highlighted here, there is some small-scale clinical evidence suggesting that meditation techniques with integrated mindfulness components may induce psychotic episodes in individuals with and without a psychiatric history. However, this evidence stems from case reports with very low participant numbers (including single cases) and relates to individuals that have engaged in intensive meditation retreats. For the participants of these 
studies, practicing meditation for up to 18 hours per day under conditions of silence and/or fasting most likely represented an abrupt alteration to their daily routine. Within the Buddhist meditative tradition in particular, a philosophy of quality and not quantity of meditation is widely advocated, and practicing meditation in an extreme and potentially stressful manner is discouraged (Shonin et al., 2013b). This accords well with the clinical consensus that stress is influential in the onset of psychosis (Brown et al., 2010). Therefore, irrespective of the participant's clinical history, it is perhaps unsurprising that intensive meditation practice precipitated psychotic episodes in some individuals.

Furthermore, the uncontrolled nature of these studies makes it difficult to conclusively pinpoint mindfulness (i.e., as opposed to other meditation techniques or environmental stressors such as lack of food, etc.) as the source of the psychotic episodes. This is a particularly important because numerous reports of adverse effects exist for non-mindfulness variants of meditation such as Transcendental Meditation and Qigong. Examples are panic attacks, musculoskeletal pain, addiction (i.e., to meditation), anti-social behaviour, impaired reality testing, dissociation, guilt, uncomfortable kinaesthetic sensations, despair, suicidal feelings, and exhaustion (Perez-De-Albeniz \& Holmes, 2000; Yorston, 2001). Therefore, whilst techniques such as mindfulness meditation, Transcendental Meditation, and Qigong can be broadly grouped together as modalities of 'meditation', it is important to highlight that these techniques reflect fundamentally different approaches. For example, Transcendental Meditation is a commercial technique introduced in the 1950s by Maharishi Mahesh Yogi that includes mantra recitation and derives from Hinduism. Conversely, mindfulness meditation is a 2,500 year old Buddhist practice that does not include chanting or mantra recitation but primarily focusses on breath and present-moment awareness. 
Although small in quantity $(n=11)$ and not without their (previously outlined) limitations, comparatively speaking, studies that indicate an application for mindfulness meditation in the treatment of psychosis appear to constitute a more reliable empirical evidence base. Furthermore, in addition to the more methodologically robust designs of these studies (i.e., controlled studies, RCTs), they were transparent in terms of whether non-mindfulness-based meditative techniques were also practiced.

Unlike more analytical meditative methods (that belong to a form of meditation known as vipassana), mindfulness meditation constitutes a more passive procedure. This means that on a meditation continuum with poles of analytical meditation and concentrative meditation, mindfulness would sit somewhere in middle. The attentional set engaged during mindfulness practice is one of open acceptance to the present moment, and concentration is invariably 'anchored' to the present moment by meditatively attending to the natural flow of the in and out breath. This combined attentional strategy (i.e., non-judgemental open awareness anchored by breath observance) has not only been shown to increase perceptual distance from cognitive and affective processes, but also begets reductions in stress due to decreased autonomic and psychological arousal (Shonin et al., 2013a). Therefore, for individuals experiencing psychosis, mindfulness may confer the capacity to: (i) "allow unpleasant psychotic experiences to come into awareness", (ii) avert a conflict-inducing response (e.g., avoidance strategies, negative self-judgement, dysphoric moods, etc.), and (iii) accept both the psychotic experience and oneself (Chadwick et al., 2009, p. 410).

It is concluded that mindfulness meditation, when delivered within a clinical framework setting, appears to have a beneficial role to play in the treatment of psychosis. However, consistent with the extant empirical data relating to the clinical utility of MBTs for treating various other psychopathologies (i.e., substance use disorders, post-traumatic stress disorder, 
behavioural addictions, eating disorders, etc.), these promising findings are not yet sufficient to demonstrate a clear treatment effect for MBTs. Thus, taking into account the risks associated with other (i.e., non-mindfulness) meditational modalities, and given the highly vulnerable nature of the patient group concerned (i.e., people with psychosis), both caution and further controlled empirical investigation utilising larger samples are clearly required.

In order to mitigate the risk of mindfulness meditation actually inducing psychotic episodes (or other deleterious symptoms), the following recommendations are proposed for delivering MBIs to people with psychosis: (i) utilise short duration guided meditations (i.e., $\leq 15$ minutes), (ii) avoid prolonged periods of silence, (iii) provide additional instruction in basic anchoring techniques (e.g., counting the breath, body scanning, etc.), (iv) use smaller group sizes (e.g., $\leq 10$ participants per facilitator) with time for one-to-one discussion where required, (v) avoid the use of explicitly analytical/insight-based (i.e., vipassana) meditation techniques, (vi) employ facilitators/clinicians with at least three-years of supervised mindfulness practice and teaching experience (i.e., as opposed to current practice where clinicians sometimes have as little as one year's mindfulness practice/teaching experience following completion of a single eight-week course [Shonin et al., 2013a]), and (vii) utilise treatments of greater length than those reviewed in this paper (which ranged from just four to twelve weeks) for individuals with persistent/severe symptoms. 


\section{References}

Abba N, Chadwick P and Stevenson C (2008) Responding mindfully to distressing psychosis: A grounded theory analysis. Psychotherapy Research 18(1): 77-87.

Brown LF, Davis LW, LaRoccod VA, et al. (2010) Participant perspectives on mindfulness meditation training for anxiety in schizophrenia. American Journal of Psychiatric Rehabilitation 13(3): 224-242.

Chadwick P, Hughes S, Russell D, et al. (2009) Mindfulness groups for distressing voices and paranoia: A replication and randomised feasibility trial. Behavioural and Cognitive Psychotherapy 37(4): 403-412.

Chadwick P, Newman-Taylor K and Abba NJ (2005) Mindfulness groups for people with psychosis. Behavioural and Cognitive Psychotherapy 33(3): 351-359.

Chan-ob T and Boonyanaruthee V (1999) Meditation in association with psychosis. Journal of Medical Association of Thailand 82(9): 925-929.

Chien WT and Lee IY (2013) The mindfulness-based psychoeducation program for Chinese patients with schizophrenia. Psychiatric Services 64(4): 376-379. 
Dennick L, Fox AP and Walter-Brice A (2013) Mindfulness groups for people experiencing distressing psychosis: An interpretative phenomenological analysis. Mental Health Review Journal 18(1): 32-43.

Garcia-Trujillo R, Monterrey AL and Gonzalez de Riviera JL (1992) Meditacion y psicosis. Psiquis Revista de Psiquiatria Psicologia y Psicosomatica 13(6-7): 39-43.

Jacobsen P, Morris E, Johns L, et al. (2011) Mindfulness groups for psychosis; key issues for implementation on an inpatient unit. Behavioural and Cognitive Psychotherapy 39(3): $349-53$.

Kuijpers HJH, van der Heijden FMMA, Tuinier S, et al. (2007) Meditation-induced psychosis. Psychopathology 40(6): 461-464.

Langer AI, Cangas AJ, Salcedo E, et al. (2012) Applying mindfulness therapy in a group of psychotic individuals: a controlled study. Behavioural and Cognitive Psychotherapy 40(1): 105-109.

Perez-De-Albeniz A and Holmes J (2000) Meditation: concepts, effects and uses in therapy. International Journal of Psychotherapy 5(1): 49-59.

Sethi S and Subhash C (2003) Relationship of meditation and psychosis: Case studies. Australian and New Zealand Journal of Psychiatry 37(3): 382.

Shonin E, Van Gordon W and Griffiths MD (2013a) Mindfulness-based interventions: Towards mindful clinical integration. Frontiers in Psychology 4(194): Doi: 10.3389/fpsyg.2013.00194. 
Shonin E, Van Gordon W and Griffiths MD (2013b) Buddhist philosophy for the treatment of problem gambling. Journal of Behavioral Addictions 2(2): 63-71.

Taylor KN, Harper S and Chadwick P (2009) Impact of mindfulness on cognition and affect in voice hearing: Evidence from two case studies. Behavioural and Cognitive Psychotherapy 37(4): 397-402.

van der Valk R, van de Waerdt S, Meijer CJ, et al. (2013) Feasibility of mindfulness-based therapy in patients recovering from a first psychotic episode: A pilot study. Early Intervention Psychiatry 7(1): 64-70.

Walsh R and Roche L (1979) Precipitation of acute psychotic episodes by intensive meditation in individuals with a history of schizophrenia. American Psychiatry Association 136(8): 1085-1086

York M (2007) A qualitative study into the experience of individuals involved in a mindfulness group within an acute inpatient mental health unit. Journal of Psychiatric and Mental Health Nursing 14(6): 603-608.

Yorston G (2001) Mania precipitated by meditation: A case report and literature review. Mental Health, Religion and Culture 4(2): 209-213. 


\section{Declaration of Interests}

The authors report no conflicts of interest. The authors alone are responsible for the content and writing of the paper. 